\title{
Detachment in fusion plasmas with symmetry breaking magnetic perturbation fields
}

\author{
H. Frerich» ${ }^{*}$ and O. Schmitz \\ Department of Engineering Physics, University of Wisconsin - Madison, WI 53706, USA \\ X. Bonnin and A. Loarte \\ ITER Organization, Route de Vinon-sur-Verdon, \\ CS 90 046, 13067 St Paul Lez Durance Cedex, France \\ Y. Feng \\ Max-Planck-Institut für Plasmaphysik, Association EURATOM-IPP, 17491 Greifswald, Germany \\ L. Li \\ College of Science, Donghua University, Shanghai 201620, People's Republic of China \\ Y. Q. Liu \\ General Atomics, PO Box 85608, San Diego, CA 92186-5608, USA \\ D. Reiter \\ Institute for Laser and Plasma Physics, Heinrich-Heine-University, D-40225 Duesseldorf, Germany
}

(Dated: September 9, 2020)

\begin{abstract}
Power exhaust from the bulk plasma is significantly altered by symmetry breaking magnetic perturbation fields, because these create direct connections (perturbed field lines) from the confined high temperature plasma to solid surfaces. The same amount of power is distributed among those new exhaust channels as for a symmetric magnetic configuration, which reduces the local upstream heat flux flowing down the perturbed field lines, and thereby making access to detachment easier (i.e. at lower upstream density) for the divertor plasma near the location corresponding to the symmetric magnetic separatrix. However, the divertor plasma regions with connection to the bulk plasma are extended non-axisymmetrically further outside, where significant heat loads occur unlike in the symmetric configuration. The temperature remains high at those locations which reduces the divertor plasma dissipation capacity making the mitigation of heat loads more difficult to achieve.
\end{abstract}

Developing a viable solution for the plasma boundary remains a grand challenge for magnetically confined fusion energy production, because it involves the interplay of a number of fundamentally different, but individually highly non-linear processes. The neutral particles in the surrounding of the plasma - and in particular the ones originating in the neutralization and re-emission process of plasma ions striking material surfaces (a.k.a. recycling) - provide an energy sink to the boundary plasma. This sink can be highly localized with a strongly varying energy loss rate depending on the local plasma temperature. When the plasma density is increased to levels relevant for later high fusion energy gain, the plasma temperature in front of material surfaces can be reduced, thereby rapidly crossing through a regime in which the energy loss rates change over several orders of magnitude for comparably small (several $\mathrm{eV}$ ) changes in the plasma temperature. This mechanism is advantageous, because the conductive energy out-flux is mostly exhausted through interaction with neutral particles and/or seeded impurities, while the remaining energy and also particle flux to dedicated material surfaces (divertor targets) is significantly reduced. This non-linear energy loss scenario is called detachment 1, 2]. In ITER (the next step magnetically confined fusion energy experiment based on the axisymmetric tokamak concept 3 ), it has to be combined with the challenge of suppressing a typical class of pressure gradient and current density driven edge instabilities, the edge localized modes (ELMs) 4], which are naturally associated with standard high confinement (Hmode) operation [5. ELM control may be achieved by introducing three-dimensional (3D), chaotic magnetic field structures from small amplitude, symmetry breaking external magnetic perturbation (MP) fields [6] 8]. In this paper, we extend an earlier exploration of the linear recycling regime with MP fields 9] into the non-linear regime, and evaluate for the first time the impact of MP fields on detachment in ITER (which is the foreseen divertor operational regime). We highlight changes in divertor operation resulting from connecting the bulk plasma to strike locations in both the nominal near and far scrapeoff layer (SOL). For this we focus on the initial H-mode plasmas to be obtained in the Pre-Fusion Plasma Operation (PFPO) phase with up to $30 \mathrm{MW}$ of additional heating for which no active measures to mitigate divertor head loads are expected to be required. We leave the added complexity of supplementing and fine tuning edge radiation by impurity seeding at the $Q=10$ heating power $(50 \mathrm{MW}$ of additional heating plus $100 \mathrm{MW}$ of alpha heating) for a later, more detailed analysis. 
a)

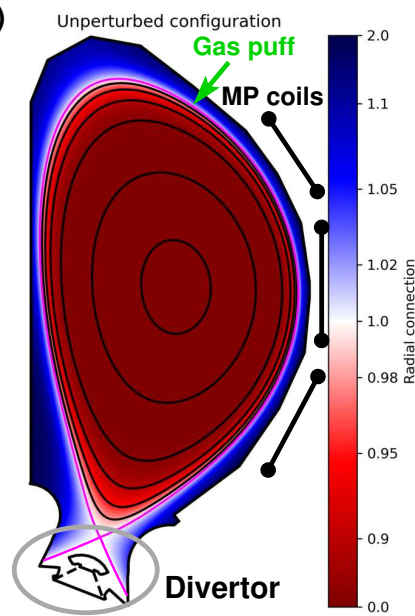

b)

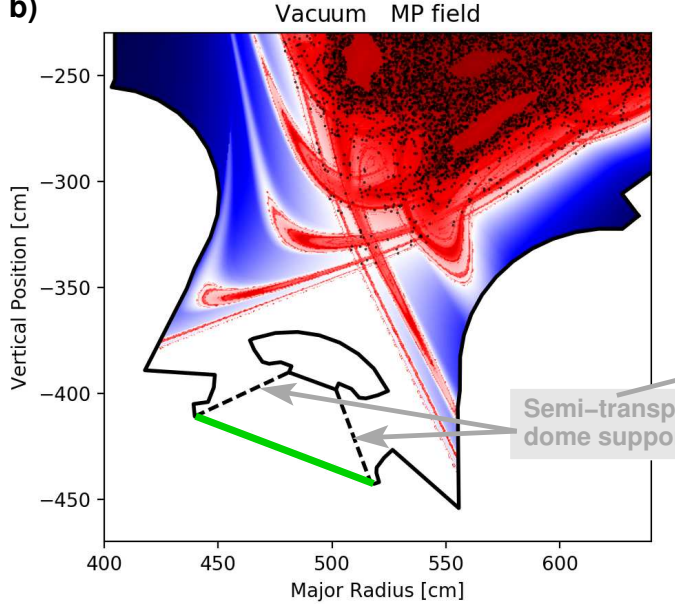

c)

Plasma response MP field

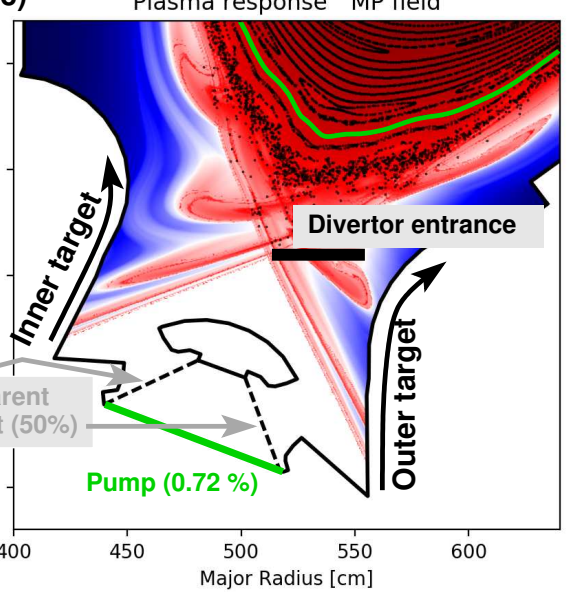

FIG. 1. Magnetic geometry of an unperturbed (axisymmetric) configuration $\mathbf{B}_{\text {equi }}$ anticipated for the PFPO phase in ITER running at low power $(30 \mathrm{MW})$ and reduced field/current $(1.8 \mathrm{~T} / 5 \mathrm{MA})$. (b) Perturbed configuration $\mathbf{B}_{\text {equi }}+\mathbf{B}_{\mathrm{MP}}$ with external MP field powered by $30 \mathrm{kA}$ coil current in $n=3$ toroidal mode number configuration and phasing optimized for ELM control. (c) Perturbed configuration $\mathbf{B}_{\text {equi }}+\mathbf{B}_{\mathrm{MP}}+\mathbf{B}_{\text {plasma }}$ including the internal response to the MP field calculated by MARS-F. A Poincaré map is shown by black dots, and the radial connection $\mathcal{R}$ (evaluated as the minimum of the normalized poloidal magnetic flux $\Psi_{N}$ that a perturbed field line encounters) is shown in color.

The unperturbed (axisymmetric) configuration $\mathbf{B}_{\text {equi }}$ is shown in figure 1 (a) for reference: the bulk plasma with closed, nested magnetic flux surfaces $\Psi_{N}$ is disconnected by a magnetic separatrix (purple) from the scrapeoff layer (SOL) where field lines are diverted onto dedicated targets. During application of small perturbations $\mathbf{B}_{\mathrm{MP}}$, a chaotic layer is formed by overlap of neighboring magnetic island chains that appear where $\mathbf{B}_{\mathrm{MP}} \cdot \nabla \Psi_{N}$ is resonant with the field line pitch angle of the helical $\mathbf{B}_{\text {equi }}$. Furthermore, the magnetic separatrix splits into two distinct (forward and backward) branches of helical lobes which are determined by the non-resonant components [10] (see e.g. figure 1 in Ref. 11). This structure is not specific to magnetic confinement fusion plasmas, but is a rather well known phenomenon in non-linear dynamics in perturbed systems with a hyperbolic fixed point [12, 13]. Field lines from the chaotic layer can escape to the divertor targets guided by this perturbed nonaxisymmetric separatrix. The magnetic geometry can be modified by an internal response $\mathbf{B}_{\text {plasma }}$ of the plasma to the external $\mathbf{B}_{\mathrm{MP}}$ (see e.g. Ref. 14 for a brief review of present magnetohydrodynamics (MHD) models for plasma response). In this paper, we take into account such a response by applying MARS-F [15]: a linearized, resistive single fluid MHD model.

In the following we focus on MP application in ITER with toroidal mode number $n=3$, and select one specific case for which the phasing between the 3 rows of windowframe type coils has been optimized for ELM control (by maximizing the kink-peeling amplification near the separatrix) [16. We find that the plasma response combines strong screening of the resonances in the bulk plasma with an amplification of mostly non-resonant components at the plasma edge just inside the separatrix of the symmetric configuration. The former is reflected in Poincaré plots, which show that the resulting chaotic domain in figure 1 (c) with $\mathbf{B}_{\text {plasma }}$ included is smaller than in (b) where $\mathbf{B}_{\text {plasma }}$ has been neglected. The latter is reflected in the colored contours which show that the radial extension of the helical lobe structure of the perturbed separatrix is similar in both cases and does not shrink as expected from screening-only models (which seem to fit well for ASDEX Upgrade [17]). It should be noted that this model (MARS-F) is capable of quantitatively re-producing the measured 3D plasma response field in DIII-D plasmas and predicting the optimal RMP coil current phasing in MAST (see Ref. 14 and Refs. therein) even though MP penetration is a highly non-linear process [18. Also, it should be noted that pedestal conditions in ITER and present day machines are not the same which may be responsible for different levels of amplification.

Fluid models are commonly applied for characterization of the plasma boundary by local parameters such as the plasma density $n$, flow velocity along field lines $u_{\|}$, and temperature $T$ (often split into an electron and ion component $\left.T_{e}, T_{i}\right)$. In steady state, fluid equations are of the form

$$
\begin{aligned}
\nabla \cdot \boldsymbol{\Gamma} & =S_{p} \\
\nabla \cdot \mathcal{M} & =-\nabla_{\|} p+S_{m} \\
\nabla \cdot \mathbf{q} & =S_{e}
\end{aligned}
$$

where particle flux $\boldsymbol{\Gamma}$ is balanced by sources/sinks $S_{p}$ (from ionization of neutral particles or recombination). 

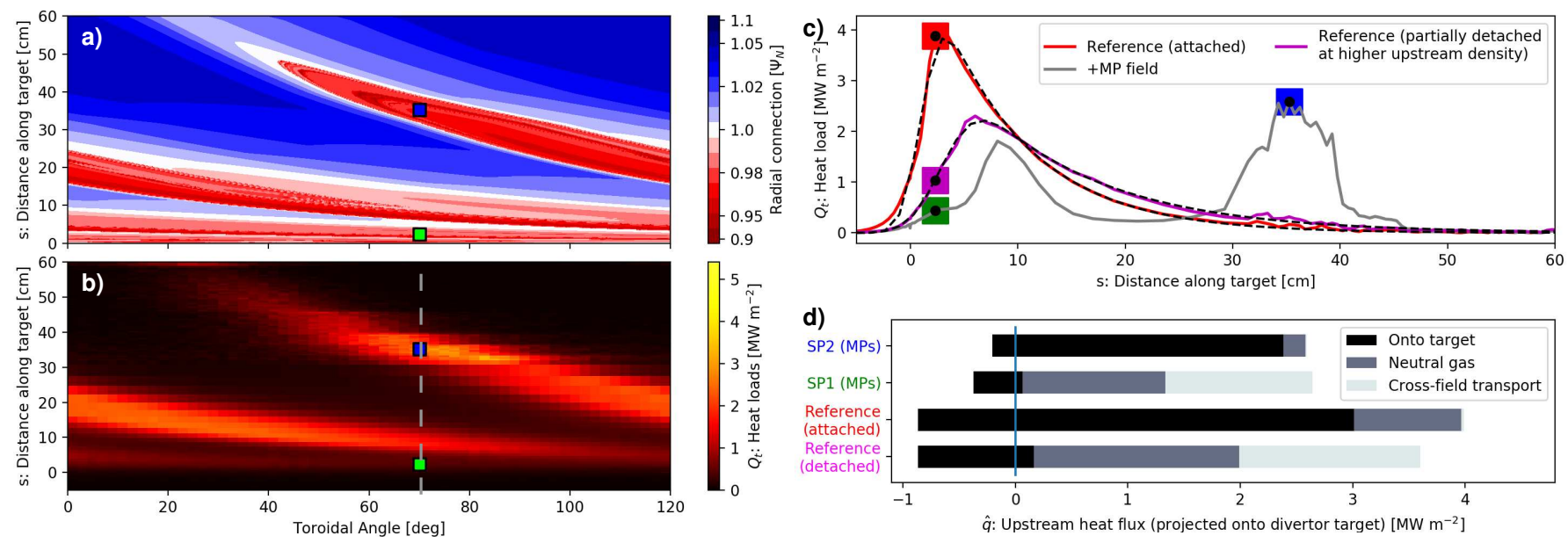

FIG. 2. (a) Magnetic footprint at the outer divertor target (OT) in figure 1 (c), characterized by the radial connection $\mathcal{R}$ of field lines. (b) Corresponding heat loads $Q_{t}$ as calculated by EMC3-EIRENE for $\Gamma_{\text {gas puff }}=3 \cdot 10^{22} \mathrm{~s}^{-1}$. Profiles of $Q_{t}$ at the highlighted toroidal position are shown in (c) in comparison to the (unperturbed) reference configuration with a fit (dashed line) to eq. 2 in Ref. [19. The distance along the target is measured from the symmetric magnetic separatrix strike point. (d) Power balance on field line at selected strike locations: the upstream heat flux $\hat{q}$ is either deposited on the target (black), dissipated from interaction with neutral gas (dark-gray) or spread through cross-field transport (light-gray). The negative extension of the black bars indicate the contribution of surface recombination to $Q_{t}$ which is not provided by $\hat{q}$.

Momentum flux $\mathcal{M}$ along field lines is driven by a gradient of the plasma pressure $p=n\left(T_{e}+T_{i}\right)$ and is balanced by momentum losses $S_{m}$ (largely from charge exchange with neutral particles), and heat flux $\mathbf{q}$ is balanced by energy losses $S_{e}$ (from ionization and molecular dissociation, including excitation and subsequent photon emission). Unlike common fluids however, transport is extremely anisotropic in plasmas, and transport along field lines exceeds cross-field transport by several orders of magnitude. The former is represented reasonably well within the classical closure of the fluid equations for collisional plasmas [20, which results in conductive heat fluxes

$$
q_{\|}^{\text {(cond.) }}=-\kappa_{\|} \nabla_{\|} T, \quad \kappa_{\|} \sim T^{5 / 2}
$$

with strong non-linear conductivity $\kappa_{\|}$. The latter is less well understood, and is in macroscopic models of the plasma boundary commonly referred to as anomalous transport to be described by a diffusion ansatz with free model parameters. The design of the ITER divertor has been guided by a two-dimensional (axisymmetric) implementation of (1)-(3), namely SOLPS [21, but whether detachment remains compatible with MP application has not been addressed. This requires a 3D model instead (such as EMC3-EIRENE [22]). After recent improvements regarding stabilization of the iterative approximation of the non-linear plasma boundary for low divertor temperatures, numerical access to detached plasmas in non-axisymmetric tokamak configurations is now possible for the first time 23]. Simulations are performed based on a standard set of model parameters for cross- field diffusion $\left(D_{\perp}=0.3 \mathrm{~m}^{2} \mathrm{~s}^{-1}\right.$ and $\left.\chi_{\perp}=1 \mathrm{~m}^{2} \mathrm{~s}^{-1}\right)$, which results in an upstream (near-SOL) power fall-off length $\lambda_{q} \approx 3-4 \mathrm{~mm}$ consistent with figure 4 in Ref. 24. Presently, $\mathbf{E} \times \mathbf{B}$ drifts are not included in the $3 \mathrm{D}$ model (and were omitted in most 2D simulations guiding the ITER divertor design as well). Steady state particle balance is maintained between gas puffing ( $\left.\Gamma_{\text {gas puff }}\right)$, fueling at the core boundary $\left(\Gamma_{\text {core }}=5 \cdot 10^{20} \mathrm{~s}^{-1}\right)$ just inside the last closed flux surface, and pumping from below the dome with a probability of $0.72 \%$ (locations are indicated in green in figure 1). The edge heating power is set to $P_{\mathrm{SOL}}=30 \mathrm{MW}$ for the PFPO phase.

A key role for the divertor state is the boundary condition for the plasma due to interaction with solid surfaces. Macroscopic plasma models do not resolve the thin plasma sheath in front of solid surfaces, but rather link the incoming heat flux $q_{t}$ to the particle flux $\Gamma_{t}$ through a sheath heat transmission coefficient $\gamma \sim 7$ and the plasma temperature $T_{t}$ :

$$
q_{t}=\gamma T_{t} \Gamma_{t}, \quad Q_{t}=q_{t}+\varepsilon \Gamma_{t},
$$

thereby eliminating 1 degree of freedom between the 3 parameters $\Gamma_{t}, T_{t}$ and $q_{t}$. The resulting heat load on the target $Q_{t}$ includes the deposited energy $\varepsilon \approx 13.6+2 \mathrm{eV}$ from recombination of the incident ion flux into atoms and further into molecules. Power dissipation in the divertor can be evaluated following the two point formatting (section 11 in Ref. 2) by mapping the heat flux $q_{u \|}$ at the divertor entrance upstream (evaluated just above the $\mathrm{X}$-point) along field lines to the target: 


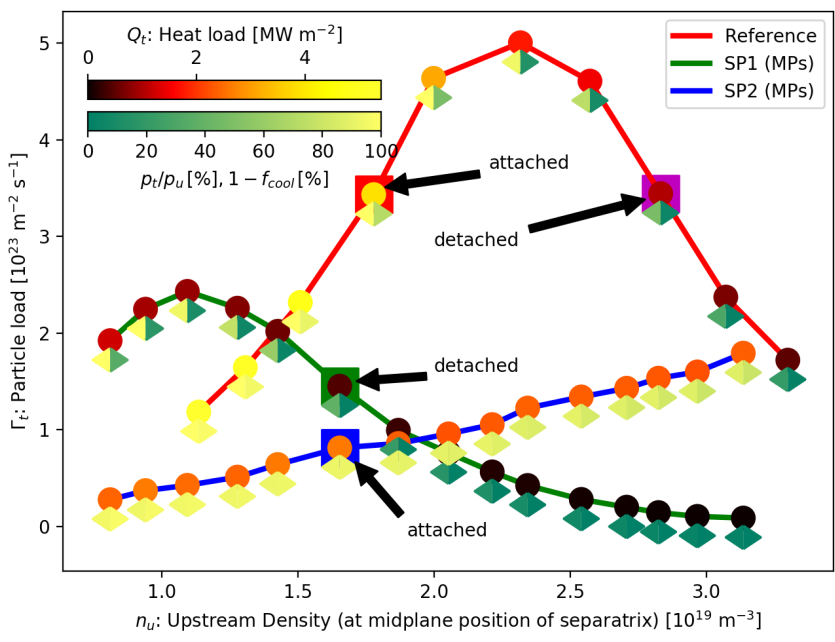

FIG. 3. Characteristic curves of the divertor state with and without MP field. Corresponding heat loads $Q_{t}$ are shown by colored circles, while pressure $(\triangleleft)$ and power balances $(\bullet)$ are shown by colored symbols below. Results from figure 2 (c) and (d) are highlighted by colored boxes and are annotated with arrows.

$$
1-f_{\text {cool }}=\frac{q_{t \|}}{B_{t}} \frac{B_{u}}{q_{u} \|}=q_{t}(\underbrace{q_{u \|} \frac{B_{t}}{B_{u}} \sin \theta}_{=\hat{q}})^{-1}
$$

where $q_{t}=q_{t \|} \sin \theta$ accounts for the oblique incident of field lines on the target.

In comparing figures 2 (a) and (b), one can see that heat loads appear where perturbed field lines connect into the bulk plasma. Figure 2 (c) shows that the unperturbed configuration (red) is still attached in a simulation with comparable upstream density $n_{u} \approx 1.6-1.7 \cdot 10^{19} \mathrm{~m}^{-3}$ (see also figure 3), and that the profile matches the archetypal shape of a convolution of an exponential with a Gaussian function (black dashed line, obtained by fitting simulation results to equation 2 in Ref. (19). The corresponding power balance in figure 2 (d) shows that most of the upstream heat flux $\hat{q}$ is indeed deposited on the target while only a minor part is dissipated through interaction with neutral gas. Integrated over the nearSOL (up to $s \approx 10 \mathrm{~cm}$ ), $65 \%$ of the power entering the divertor upstream is still deposited on the target. A partially detached state (purple) at higher upstream density is shown for comparison, and here the majority of $\hat{q}$ is either dissipated or spread through cross-field transport (note that the profile shape in figure 2 (c) is still represented by eq. 2 in Ref 19, but that it is shifted further outwards with respect to the attached case).

In the following we compare divertor performance at two strike points with magnetic connection to the bulk plasma when MP fields are applied: SP1 located in the former near-SOL (highlighted in green in figure 2), and
SP2 located in the former far-SOL (highlighted in blue). The reference for SP1 is highlighted red in figure 2 (c), while the reference divertor state for SP2 is irrelevant because $\hat{q}$ is negligible here. The power balance in figure 2 (d) shows that SP1 is already detached, similar to the detached reference case without MP fields at higher $n_{u}$. Integrated over the near-SOL, only $14 \%$ of the upstream influx is deposited on the target. SP2, however, remains attached with significant upstream heat flux $\hat{q}$ being deposited onto the divertor target without dissipation.

A density scan (by varying $\Gamma_{\text {gas puff }}$ ) has been performed to explore the impact of MP fields on the transition to detachment, and the resulting characteristic curves for $\Gamma_{t}$ are shown in figure 3 . The unperturbed SP (red line) exhibits the typical behavior: particle loads are initially increasing and then come to a roll-over point (detachment transition) once power losses $\left(f_{\text {cool }}\right)$ set in. After the roll-over, the detached state is characterized by both pressure and power losses between the divertor entrance and the target. The same behavior is found with MP field at SP1 (green line), except roll-over occurs already at a lower density with lower peak particle load. This is completely different at SP2 (blue line) which remains attached at high $T_{t}$, and the resulting high $Q_{t}$ ultimately exceeds that of the unperturbed reference once the latter detaches. The higher $T_{t}$ at SP2 follows from:

1. higher upstream temperature $T_{u}$ due to a deeper connection into the bulk plasma evident from figure 2 (a), and

2. a shorter connection length to the bulk plasma (resulting from field lines passing the X-point at a greater distance) causing a tighter coupling between $T_{u}$ and $T_{t}$ according to (4).

This is possibly reinforced by the divertor target geometry which favors reflection of recycled neutral particles away from the far-SOL, thereby reducing their contributions to dissipation here and keeping SP2 in a lowrecycling state (unlike in open divertor configurations such as in DIII-D where the opposite trend is observed [25, 26]). Power losses are in fact negligible at SP2 $\left(f_{\text {cool }} \approx 0\right.$, see figure 3$)$, thereby leading to a linear decrease of $T_{t}$ with the inverse of $\Gamma_{t}$ as long as $\hat{q}$ (and thus $q_{t}$ ) remains constant. A moderate increase of $q_{t}$ may even be found at SP2 from re-directing power exhaust from the bulk plasma during the detachment transition at SP1. The presence of such a regime was identified by experimental results at the EAST tokamak [27. It is important to note that even higher $T_{u}$ is expected for the full power burning plasma phase, which implies even more severe conditions at the far-SOL strike locations connected to the bulk plasma, and thus raises concerns regarding the magnitude of the local power fluxes and divertor lifetime (tungsten sputtering). To address these concerns, the ITER ELM control coil system is designed 
to apply rotating MP fields at frequencies of several $\mathrm{Hz}$ 28]; however it is desirable to use this capability only when needed to minimize fatigue lifetime consumption of these coils. To determine when such capability will be needed, it is necessary to further develop and apply high fidelity models for the perturbed plasma boundary (including effects of plasma response, impurity seeding, $\mathbf{E} \times \mathbf{B}$ drifts, ...), and to further validate their predictions experimentally.

Conclusions: Power exhaust from the bulk plasma is significantly altered by MP fields due to direct connections (i.e. transport along field lines) to both near-SOL and far-SOL strike locations on the divertor targets. The same amount of $P_{\mathrm{SOL}}$ is distributed among those exhaust channels, leading to lower near-SOL $\hat{q}$ at comparable upstream densities, and thereby making detachment transition easier at these locations. Conversely, $\hat{q}$ to the farSOL is significantly increased, and temperatures remain higher due to the more direct link to the bulk plasma. Thus, unlike in the reference configuration, a later detachment of the far-SOL implies significant heat loads to those locations which are more difficult to mitigate on the divertor targets at ITER.

\section{ACKNOWLEDGEMENTS}

This work was supported by the U.S. Department of Energy under grants DE-SC0020357, DE-SC00013911 and DE-SC0020284, and by the College of Engineering at the University of Wisconsin - Madison. This work was done under the auspices of the ITER Scientist Fellow Network. The views and opinions expressed herein do not necessarily reflect those of the ITER Organization. Computer simulations have been performed at the UW-Madison Center for High Throughput Computing (CHTC) and at the ITER HPC cluster.

* hfrerichs@wisc.edu

[1] S. I. Krasheninnikov and A. S. Kukushkin. Physics of ultimate detachment of a tokamak divertor plasma. $J$. Plasma Phys., 83(5):155830501, 2017.

[2] P. C. Stangeby. Basic physical processes and reduced models for plasma detachment. Plasma Phys. Control. Fusion, 60:044022, 2018.

[3] J. Wesson. Tokamaks, 4th edition, volume 149 of International Series of Monographs on Physics. Oxford University Press, 2011.

[4] H. Zohm. Edge localized modes (ELMs). Plasma Phys. Control. Fusion, 38:105-128, 1996.

[5] ITER Physics Expert Group on Confinement and Transport. Chapter 2: Plasma confinement and transport. Nuclear Fusion, 39:2175, 1999.

[6] T. E. Evans, R. A. Moyer, P. R. Thomas, J. G. Watkins, T. H. Osborne, J. A. Boedo, E. J. Doyle, M. E. Fen- stermacher, K. H. Finken, R. J. Groebner, M. Groth, J. H. Harris, R. J. La Haye, C. J. Lasnier, S. Masuzaki, N. Ohyabu, D. G. Pretty, T. L. Rhodes, H. Reimerdes, D. L. Rudakov, M. J. Schaffer, G. Wang, and L. Zeng. Suppression of Large Edge-Localized Modes in HighConfinement DIII-D Plasmas with a Stochastic Magnetic Boundary. Phys. Rev. Lett., 92(23):235003, 2004.

[7] Y. Liang, H. R. Koslowski, P. R. Thomas, E. Nardon, B. Alper, P. Andrew, Y. Andrew, G. Arnoux, Y. Baranov, M. Becoulet, M. Beurskens, T. Biewer, M. Bigi, K. Crombe, E. De La Luna, P. de Vries, W. Fundamenski, S. Gerasimov, C. Giroud, M. P. Gryaznevich, N. Hawkes, S. Hotchin, D. Howell, S. Jachmich, V. Kiptily, L. Moreira, V. Parail, S. D. Pinches, E. Rachlew, and O. Zimmermann. Active Control of Type-I EdgeLocalized Modes with $\mathrm{n}=1$ Perturbation Fields in the JET Tokamak. Phys. Rev. Lett., 98(265004):1-5, 2007.

[8] W. Suttrop, T. Eich, J. C. Fuchs, S. Günter, A. Janzer, A. Herrmann, A. Kallenbach, P. T. Lang, T. Lunt, M. Maraschek, R. M. McDermott, A. Mlynek, T. Pütterich, M. Rott, T. Vierle, E. Wolfrum, Q. Yu, I. Zammuto, H. Zohm, and ASDEX Upgrade Team. First Observation of Edge Localized Modes Mitigation with Resonant and Nonresonant Magnetic Perturbations in ASDEX Upgrade. Phys. Rev. Lett., 106:225004, 2011.

[9] O. Schmitz, M. Becoulet, P. Cahyna, T.E. Evans, Y. Feng, H. Frerichs, A. Loarte, R.A. Pitts, D. Reiser, M.E. Fenstermacher, D. Harting, A. Kirschner, A. Kukushkin, T. Lunt, G. Saibene, D. Reiter, U. Samm, and S. Wiesen. Three-dimensional modeling of plasma edge transport and divertor fluxes during application of resonant magnetic perturbations on ITER. Nuclear Fusion, 56(6):066008, 2016.

[10] T. E. Evans, R. K. W. Roeder, J. A. Carter, and B. I. Rapoport. Homoclinic tangles, bifurcations and edge stochasticity in diverted tokamaks. Contrib. Plasma Phys., 44:235-240, 2004.

[11] H. Frerichs, O. Schmitz, T. Evans, Y. Feng, and D. Reiter. The pattern of parallel edge plasma flows due to pressure gradients, recycling, and resonant magnetic perturbations in DIII-D. Phys. Plasmas, 22:072508, 2015.

[12] J. Guckenheimer and P. Holmes. Nonlinear Oscillations, Dynamical Systems, and Bifurcations of Vector Fields. Applied Mathematical Science, 1983.

[13] A. J. Lichtenberg and M. A. Lieberman. Regular and chaotic dynamics. Applied mathematical sciences 38, 2nd edition, 1992.

[14] Y. Liu, C. J. Ham, A. Kirk, L. Li, A. Loarte, D. A. Ryan, Y. Sun, W. Suttrop, X. Yang, and L. Zhou. ELM control with RMP: plasma response models and the role of edge peeling response. Plasma Phys. Control. Fusion, 58:114005, 2016.

[15] Y. Q. Liu, A. Bondeson, C. M. Fransson, B. Lennartson, and C. Breitholtz. Feedback stabilization of nonaxisymmetric resistive wall modes in tokamaks. I. Electromagnetic model. Phys. Plasmas, 7:3681, 2000.

[16] L. Li, Y. Q. Liu, N. Wang, A. Kirk, H. R. Koslowski, Y. Liang, A. Loarte, D. Ryan, and F. C. Zhong. Toroidal modeling of plasma response to RMP fields in ITER. Plasma Phys. Control. Fusion, 59:044005, 2017.

[17] D. Brida, T. Lunt, M. Wischmeier, M. Bernert, D. Carralero, M. Faitsch, Y. Feng, T. Sehmer, B. Sieglin, W. Suttrop, E. Wolfrum, The ASDEX Upgrade Team, and The MST1 Team. Heat flux pattern in detached L- 
modes and ELM mitigated H-modes with rotating magnetic perturbations in ASDEX Upgrade. Nuclear Fusion, $57: 116006,2017$.

[18] F. Orain, M. Hoelzl, F. Mink, M. Willensdorfer, M. Bécoulet, M. Dunne, S. Günter, G. Huijsmans, K. Lackner, S. Pamela, W. Suttrop, E. Viezzer, ASDEX Upgrade Team, and EUROfusion MST1 Team. Non-linear modeling of the threshold between ELM mitigation and ELM suppression by resonant magnetic perturbations in ASDEX upgrade. Phys. Plasmas, 26:042503, 2019.

[19] T. Eich, B. Sieglin, A. Scarabosio, W. Fundamenski, R.J. Goldston, A. Herrmann, and ASDEX Upgrade Team. Inter-ELM Power Decay Length for JETand ASDEX Upgrade: Measurement and Comparison with Heuristic Drift-Based Model. Phys. Rev. Lett., 107:215001, 2011.

[20] S.I. Braginskii. Transport processes in a plasma. Review of Plasma Physics, 1:205-311, 1965.

[21] A.S. Kukushkin, H.D. Pacher, V. Kotov, G.W. Pacher, and D. Reiter. Finalizing the ITER divertor design: The key role of SOLPS modeling. Fusion Eng. Des., 86:2865, 2011.

[22] Y. Feng, H. Frerichs, M. Kobayashi, and D. Reiter. Monte-Carlo fluid approaches to detached plasmas in non-axisymmetric divertor configurations. Plasma Phys. Control. Fusion, 59:034006, 2017.

[23] H. Frerichs, X. Bonnin, Y. Feng, A. Loarte, R.A. Pitts, D. Reiter, and O. Schmitz. Stabilization of EMC3EIRENE for detachment conditions and comparison to SOLPS-ITER. Nuclear Materials and Energy, 18:62-66, 2019.

[24] R.A. Pitts, X. Bonnin, F. Escourbiac, H. Frerichs, J.P.
Gunn, T. Hirai, A.S. Kukushkin, E. Kaveeva, M.A. Miller, D. Moulton, V. Rozhansky, I. Senichenkov, E. Sytova, O. Schmitz, P.C. Stangeby, G. De Temmerman, I. Veselova, and S. Wiesen. Physics basis for the first ITER tungsten divertor. Nuclear Materials and Energy, 20:100696, 2019.

[25] H. Frerichs, O. Schmitz, D. Reiter, T. E. Evans, and Y. Feng. Striation pattern of target particle and heat fluxes in three dimensional simulations for DIII-D. Phys. Plasmas, 21:020702, 2014.

[26] A.R. Briesemeister, J.-W. Ahn, J.M. Canik, M.E. Fenstermacher, H. Frerichs, C. J. Lasnier, J.D. Lore, A.W. Leonard, M.A. Makowski, A.G. McLean, W.H. Meyer, O. Schmitz, M.W. Shafer, E.A. Unterberg, H.Q. Wang, and J. G. Watkins. Changes in divertor conditions in response to changing core density with RMPs. Nuclear Fusion, 57:076038, 2017.

[27] J. Li, H. Y. Guo, B. N. Wan, X. Z. Gong, Y. F. Liang, G. S. Xu, K. F. Gan, J. S. Hu, H. Q. Wang, L. Wang, L. Zeng, Y. P. Zhao, P. Denner, G. L. Jackson, A. Loarte, R. Maingi, J. E. Menard, M. Rack, and X. L. Zou. A long-pulse high-confinement plasma regime in the Experimental Advanced Superconducting Tokamak. Nature Physics, 9:817, 2013.

[28] A. Loarte, G. Huijsmans, S. Futatani, L.R. Baylor, T.E. Evans, D. M. Orlov, O. Schmitz, M. Becoulet, P. Cahyna, Y. Gribov, A. Kavin, A. Sashala Naik, D.J. Campbell, T. Casper, E. Daly, H. Frerichs, A. Kischner, R. Laengner, S. Lisgo, R.A. Pitts, G. Saibene, and A. Wingen. Progress on the application of ELM control schemes to ITER scenarios from the non-active phase to DT operation. Nuclear Fusion, 54:033007, 2014. 